Journal of Animal and Veterinary Advances 11 (12): 2110-2115, 2012

ISSN: $1680-5593$

(C) Medwell Journals, 2012

\title{
Generate Induced Pluripotent Stem Cells by Three Factors under Feeder-Free Condition with Higher Efficiencies
}

\author{
Youde Lu, Fulu Dong, Chunlei Song, Huanhuan Wang, Changwan Du, Jing Han, \\ Ling Qin, Le Zhai, Fang Zhao, Zhourui Xie and Honglin Liu \\ Department of Animal Breeding and Genetics, College of Animal Science and Technology, \\ Nanjing Agricultural University, 210095 Nanjing, China
}

\begin{abstract}
Pluripotent stem cells can be induced from different cell types through transduction of the transcription factors by lentiviral, polycistronic vectors and other vectors. Most of these methods of generation of induced Pluripotent Stem (iPS) cells still use Mouse Embryonic Fibroblasts (MEF) as feeder layers. The use of animal feeder layers will hinder the clinical application of iPS cells because of the presence of unknown animal products, xenobiotics and when screening the intermediates iPS to study the mechanisms of cellular reprogramming, the feeder cells would be mixed in intermediates iPS cells samples. Here researchers generated iPS cells from MEF use only three factors Oct $4, \mathrm{Klf} 4$ and Sox 2 . Through add Vitamin C and gradually replaced the medium after retroviral infection, researchers promote the efficiency of induced iPS to $0.2 \%$.
\end{abstract}

Key words: Induced Pluripotent Stem (iPS) cells, MEF, feeder, feeder-free, reprogramming

\section{INTRODUCTION}

Since, Yamanaka generated induced Pluripotent Stem (iPS) cells from MEF by the transduction of four transcription factors Oct4, Sox2, c-Myc and Klf4 (Takahashi and Yamanaka, 2006). The technical of iPS cells generation have acquire a lot of achievement because of its enormous process in clinical. Various somatic cells types of different species could be used to generate iPS cells such as fibroblasts, bone marrow cells, hepatocytes and gastric epithelial cells, pancreatic cells, neural stem cells and B lymphocytes, keratinocytes and blood progenitor cells (Takahashi and Yamanaka, 2006; Aasen et al., 2008; Aoi et al., 2008; Hanna et al., 2008; Kim et al., 2008; Silva et al., 2008; Stadtfeld et al., 2008; Loh et al., 2009) and many different approaches have been used to deliver transcription factors into somatic cells such as retroviral vectors, lentiviral vectors, adenoviral vectors, Sendai virus, polycistronic minicircle vectors, self-replicating selectable episomes, purified proteins, RNA (Takahashi and Yamanaka, 2006; Okita et al., 2007; Tchieu et al., 2007; Brambrink et al., 2008; Tadtfeld et al., 2008; Fusaki et al., 2009; Loh et al., 2009; Yu et al., 2009; Zhou et al., 2009; Zhou and Freed, 2009; Jia et al., 2010; Sommer et al., 2010; Warren et al., 2010).
IPS cells technology is ultimate used for regenerative medicine, drug development. However, a majority of iPS cells generation still use MEF as feeder layers. Because there is exogenous material, zoonotic pathogens, unknown viruses in mouse feeder cells (Takahashi et al., 2009) so, it will hinder clinical translation of iPS cells and when screening the intermediates iPS cells the feeder cells would be mixed with the iPS cells samples, it would affect the study of the cellular reprogramming mechanisms and when derived and maintained under feeder conditions the iPS cells exerted differentiation variability and transcriptomic variability (Chin et al., 2009; Feng et al., 2010; Hu et al., 2010). A recent report described that feeder-free iPS cells display closer correlation with ES cells than feeder derived iPS cells (Chung et al., 2011). In the future the iPS cells which used in clinical application must be derived from feeder-free condition. Consequently, it is need to develop a method to generated iPS cells under feeder-free conditions.

Here researchers introduce a method to generate mouse iPS cells use three transcription factors Oct4, Klf4 and Sox 2 under feeder-free conditions. In previous studies, generation of iPS cells delete c-Myc from four factors would reduce the reprogramming efficiency and delay the reprogramming process (Nakagawa et al., 2008; Wernig et al., 2008) so, in this study, researchers add

Corresponding Author: Honglin Liu, Department of Animal Breeding and Genetics, College of Animal Science and Technology, Nanjing Agricultural University, 210095 Nanjing, China 
Vitamin C after virus infection and gradually replaced the medium, made the reprogramming efficiency increase to $0.2 \%$.

\section{MATERIALS AND METHODS}

Cell isolation and culture: The MEF was isolated from 13.5 day embryos of C57BL/6 mice and cultured in DMEM (high glucose) containing 10\% fetal bovine serum, NEAA, L-glutamax, sodium pyruvate and penicillin/streptomycin. ES cells and iPS cells were maintained in KnockoutDMEM supplemented with $20 \%$ Knockout Serum Replacement (KSR), $1000 \mathrm{UmL}^{-1} \mathrm{LIF}$ (Millipore), NEAA, L-glutamax, sodium pyruvate, penicillin/streptomycin and $0.1 \mathrm{mM} \beta$-mercaptoethanol under feeder condition. Unless otherwise indicated all reagents were purchased from Invitrogen.

Retroviral production and infection: The PMXS-based retroviral vectors (Addgene) encoding the mouse reprogramming genes of Klf4, Oct4, Sox 2 or EGFP were transfected into plat-E cells using Lipofectamine 2000 (Invitrogen). Virus supernatants were collected 2 days after transfection and filtered through a $0.45 \mu \mathrm{m}$ filter. MEF were seeded overnight at a density of $3500-5000$ cells cm${ }^{-2}$ and incubated with Viral-containing supernatants for Sox2, Oct4 and Klf4 (Takahashi et al., 2007). As controls MEF were incubated with GFP retrovirus alone to calculate transduction efficiencies. After $12 \mathrm{~h}$ of infection, medium was gradually changes by ES medium added $50 \mu \mathrm{g} \mathrm{mL} \mathrm{m}^{-1}$ Vitamin C (sigma) in a 4 days stepwise process (Chen et al., 2010 ; Esteban et al., 2010). For feeder iPS generation, the MEF were seeded onto 6 well plate with MEF feeder cells for feeder-free iPS cells generation the MEF were onto 6 well plate directly.

Immunocytochemistry staining: Cell samples were fixed in $3.7 \%$ paraformaldehydemn for $30 \mathrm{~min}$ at room temperature then washed with PBS 3 times and blocked with PBS containing 5\% fetal bovine serum and $0.1 \%$ Triton-X100 for $20 \mathrm{~min}$ at room temperature, cells samples were then stained with primary antibodies against Nanog and SSEA-1 for $1 \mathrm{~h}$ at room temperature, washed with PBS 3 times and incubated with anti-mouse or rabbit I gG secondary antibody for $1 \mathrm{~h}$, washed 3 times with PBS. cells were stained with DAPI for $20 \mathrm{~min}$. Nanog and SSEA1 antibodies were from Abcam (1:100 dilution), secondary antibodies were from Abcam (1:200 dilution), antibodies were diluted in $1 \%$ fetal bovine serum in PBS.
For Alkaline Phosphatase (AP) staining, cells were stained using the Alkaline Phosphatase Detection kit (Millipore).

Gene expression analysis: RNA extraction was performed using TrizoL reagent (Invitrogen) according to the protocol and reverse transcribed to first-strand cDNA from $2 \mu \mathrm{g}$ of total RNA using the M-MLV reverse transcriptase (Promega, Madison, USA) and oligo dT (18). PCR reactions were performed using the rTaq kit from Takara. The expression level of Oct4 and Rex1 were analyzed by quantitative PCR using Fast Start Universal SYBR Green Master (ROX) (Roche) and analyzed with ABI7300 fluorescence quantitative PCR instrument.

Teratomas assays: About 2 million cells were suspended in DMEM containing $10 \%$ FBS and injected subcutaneously into nude mice. Teratomas were dissected 4 weeks after injection, fixed in $4 \%$ paraformaldehyde then hematoxylin and Eosin staining was performed as per standard procedures.

Karyotype analysis: Karyotype analysis was performed as described before (Esteban et al., 2009).

\section{RESULTS AND DISCUSSION}

Generation of iPS colonies under feeder-free condition: Increasing degree of cell condensation 4 days after retroviral infection (Fig. 1a and b) and after 11 days there is many colonies with an ES cell-like morphology grew out from with or without feeder condition (Fig. 1c and d). To compare the reprogramming efficiency, researchers counted the numbers of ES-like colonies which derived with or without feeder condition. The results showed that the reprogramming efficiency of feeder iPS cells and feeder-free iPS cells was similar (Fig. 1g). It is agree to the previous study (Nakagawa et al., 2008). Through passage the feeder-free derived iPS colonies on feeder layers researchers obtain some homogeneous iPS cells lines. The feeder-free derived iPS colonies displayed similar with ES cell colonies (Fig. 1e) and was positive for Alkaline Phosphatase (AP) staining (Fig. 1f) (Fig. 1a-g).

ES marker expressed in feeder-free iPS cells: The feeder-free iPS cells were stained with Oct4, SSEA-1 and Nanog antibody to confirm whether the feeder-free iPS cells expressed ES markers. The results revealed that they are positive for Oct4, SSEA-1 and Nanog (Fig. 2a). Then researchers use RT-PCR to detect whether those iPS colonies at passage number 10 expressed ES-cell marker 

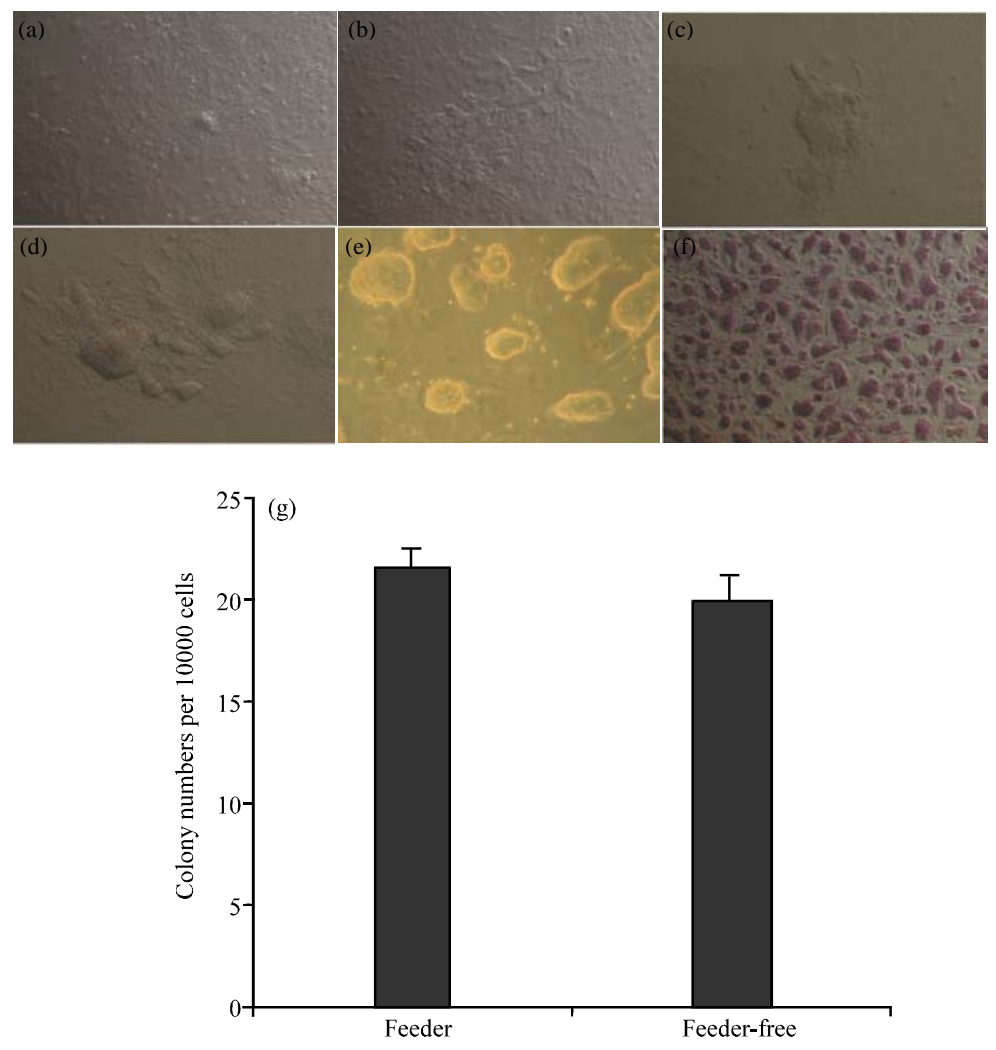

Fig. 1: About 7 days after retroviral infection cells grew a) under feeder-free conditions and b) feeder conditions. About 11 days after infection, there are many ES cell-like colonies c) under feeder-free conditions and d) feeder conditions. e) iPS cells derived feeder-free conditions and f) Alkaline Phosphatase (AP) staining for iPS cells. g) Comparison of the AP-positive colonies numbers per 10,000 cells at 14 day after retroviral infection using the feeder-free or the traditional methods

genes. The results showed that ES marker genes including Oct4, Nanog, Rex-1, Dppa5, Fgf4 and Fbx15 were expressed in those feeder-free iPS cells, feeder iPS and ES cells (Fig. 2c). Expression levels of Oct4 and Rex1 was measured by quantitative RT-PCR (Fig. 2b).

\section{The feeder-free iPS cells in vivo maintains pluripotency:}

Researchers examined the differentiation potential of feeder-free iPS cells by injecting them into nude mice to produced Teratomas (Fig. 3b). Hematoxylin and eosin staining experiment revealed that the iPS cells differentiated into all three germ layers (Fig. 3a). After 10 passages of this feeder-free iPS they also displayed a normal karyotype (Fig. 3c) and ES-like phenotype. All of these results demonstrate that the feeder-free iPS cells induced by three factors is similar to ES (Fig. $3 \mathrm{a}-\mathrm{c}$ ).

The discovery of that iPS cells can be induced from somatic cells by transduction of a few embryonic transcription factors has revolutionized the stem cell field (Takahashi and Yamanaka, 2006). Generation of iPS cells has provided a promising technology in regenerative medicine and biology, this technology overcome ethical issues and the risk of immune rejection. However, there are also many obstacles need to be overcame before clinical application of iPS cells. One of the obstacles is that the utilization of animal feeder layers in the process of generation of iPS cells. Because feeder cells would introduces unknown exogenous material/pathogens and when picked up iPS colonies the feeder cells would mixed in it. Although, feeder cells are important for maintaining the pluripotency and proliferation of iPS cells and ES cell, they are not necessary for the initiation and the process of reprogramming by four factors (Chen et al., 2009). Preparing feeder cells is tedious and need the destruction of embryo, feeder cells derived different embryo also exhibit variability use feeder layers would made the reprogramming outcomes variability, affected the consistency of iPS lines and also, introduced variable differentiation potency (Chan et al., 2009; Feng et al., 2010; Hu et al., 2010). 

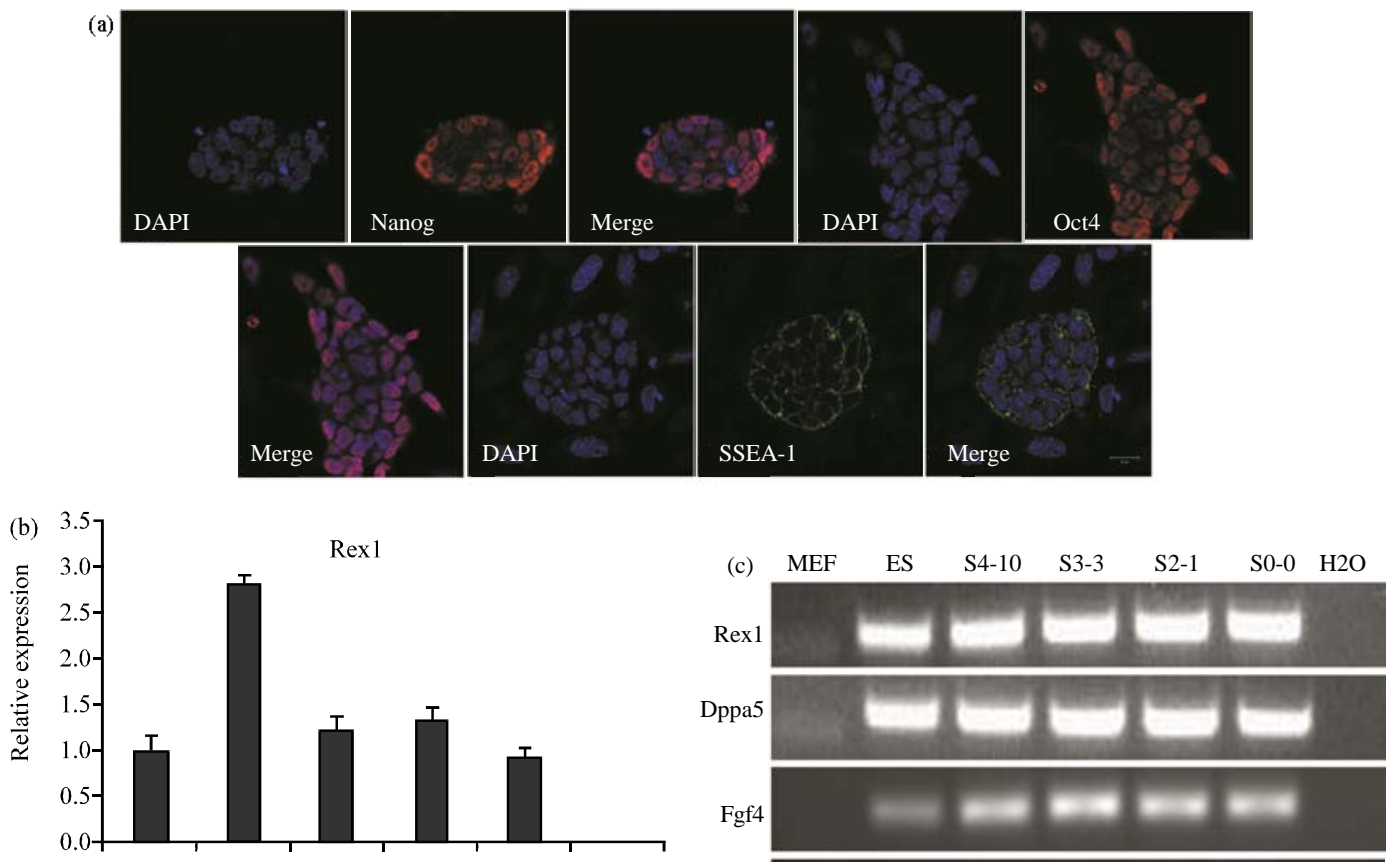

Dppa5
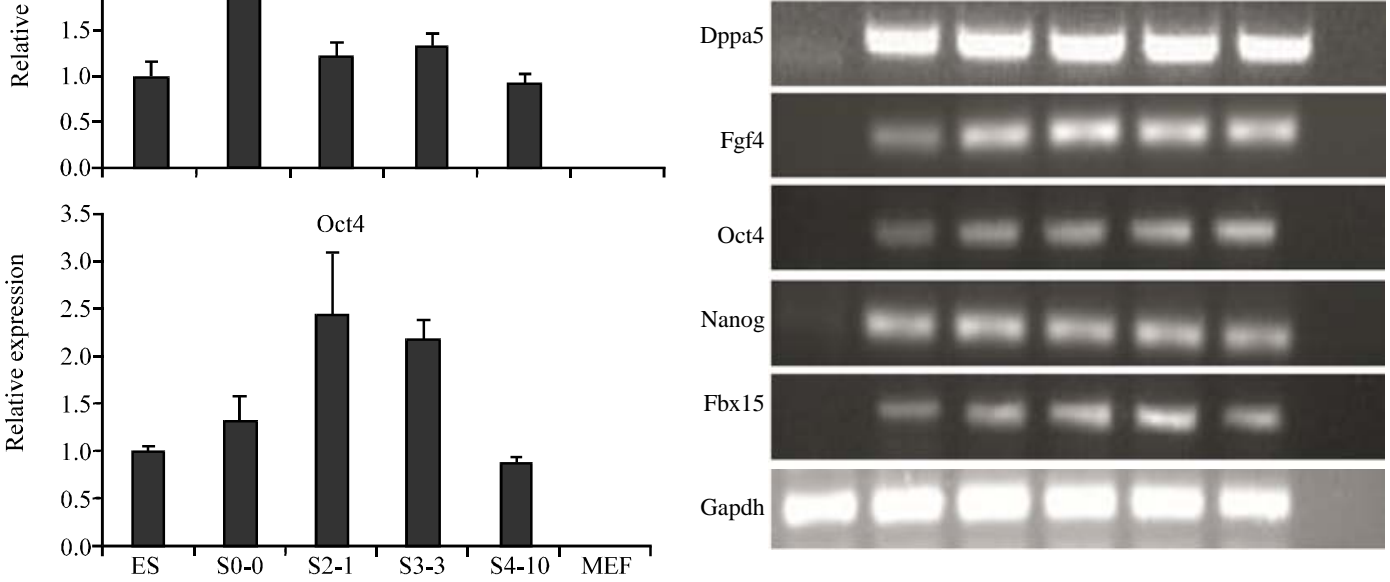

Fig. 2a: a) Immunofluorescence results for Nanog, Oct4 and SSEA-1 in feeder-free iPS cells (Scale bars $=20 \mu \mathrm{m}$ ); b) Quantitative RT-PCR results for Rex1 and Oct4 in feeder-free iPS cells (S0-0, S2-1, S0-0 and S2-1 were two different feeder-free iPS colonies) and feeder iPS cells (S3-3, S4-10, S3-3 and S4-10 were two different feeder iPS colonies); c) ES markers expressed in feeder-free iPS cells (SO-0 and S2-1) and feeder iPS cells (S3-3 and S4-10)
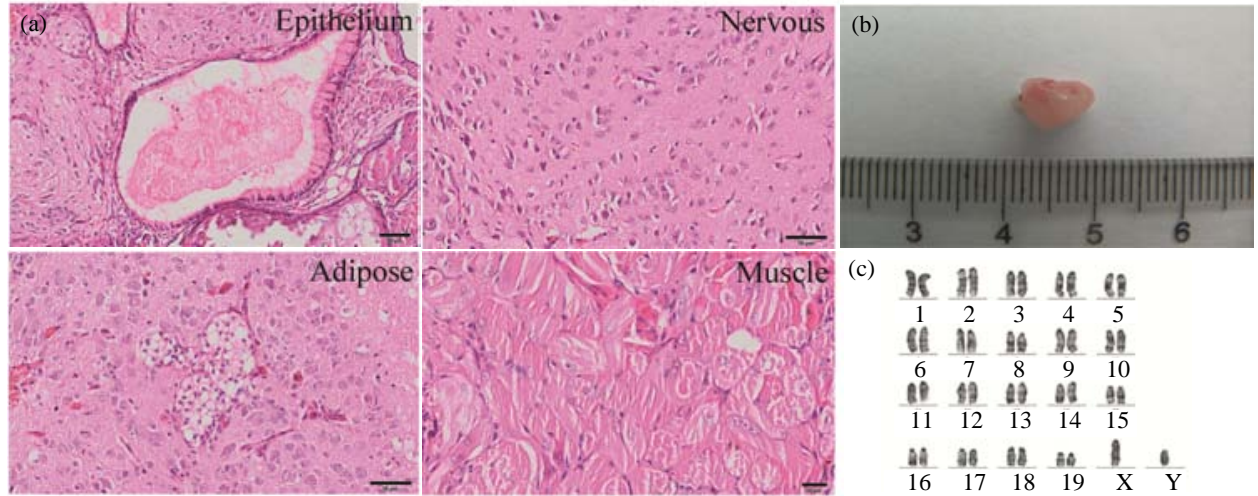

(c)

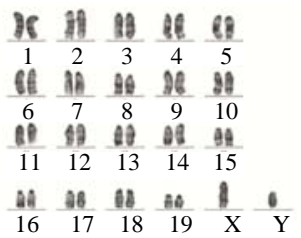

Fig. 3: a) Hematoxylin and eosin staining various tissues in teratomas from feeder-free iPS cells, epithelium, nervous, adipose (Scale bars $=50 \mu \mathrm{m}$ ) and muscle (Scale bars $=20 \mu \mathrm{m}$ ). b) Teratomas were dissected 4 weeks after injection and c) Chromosome analysis of feeder-free iPS cells after 10 passages 


\section{CONCLUSION}

In this study, researchers report that they use MEF as a model to generate feeder-free iPS cells by three factors Oct4, Sox 2 and Klf4. Through add Vitamin C and gradually replaced the medium after retroviral infection researchers promote the reprogramming efficiency to $0.2 \%$. It is not obvious disparity of reprogramming efficiency and reprogramming time between with and without feeder layers conditions. Though, iPS cells can derive from feeder-free conditions with higher efficiencies and maintain pluripotency under feeder-free conditions through supplemented with some small molecules material is also far away from clinical application. The serum containing media, transduction factors are also dangerous for clinical application. Thus, it is important to generate iPS cells in a defined and standardized system to minimize variability for clinical application.

\section{ACKNOWLEDGEMENTS}

This study was supported by the Key Project of Chinese National Programs for Fundamental Research and Development (973 Program No.: 2007CB947403) and scientific innovation research of college graduate in Jangsu province (No.: 676-2011).

\section{REFERENCES}

Aasen, T., A. Raya, M.J. Barrero, E. Garreta and A. Consiglio et al., 2008. Efficient and rapid generation of induced pluripotent stem cells from human keratinocytes. Nat. Biotechnol., 26: 1276-1284.

Aoi, T., K. Yae, M. Nakagawa, T. Ichisaka and $\mathrm{K}$. Okita et al., 2008. Generation of pluripotent stem cells from adult mouse liver and stomach cells. Science, 321: 699-702.

Brambrink, T., R. Foreman, G.G. Welstead, C.J. Lengner, M. Wernig, H. Suh and R. Jaenisch, 2008. Sequential expression of pluripotency markers during direct reprogramming of mouse somatic cells. Cell Stem. Cell, 2: 151-159.

Chan, E.M., S. Ratanasirintrawoot, I.H. Park, P.D. Manos and Y.H. Loh et al., 2009. Live cell imaging distinguishes bona fide human iPS cells from partially reprogrammed cells. Nat. Biotechnol., 27: 1033-1037.

Chen, J., J. Liu, Q. Han, D. Qin and J. Xu et al., 2010. Towards an optimized culture medium for the generation of mouse induced pluripotent stem cells. J. Biol. Chem., 285: 31066-31072.
Chen, M., X. Sun, R. Jiang, W. Shen and X. Zhong, 2009. Role of MEF feeder cells in direct reprogramming of mouse tail-tip fibroblasts. Cell Biol. Int., 33: 1268-7123.

Chin, M.H., M.J. Mason, W. Xie, S. Volinia and M. Singer et al., 2009. Induced pluripotent stem cells and embryonic stem cells are distinguished by gene expression signatures. Cell Stem Cell, 5: 111-123.

Chung, H.C., R.C. Lin, G.J. Logan, I.E. Alexander, P.S. Sachdev and K.S. Sidhu, 2011. Human induced pluripotent stem cells derived under feeder-free conditions display unique cell cycle and DNA replication gene profiles. Stem Cells Dev.

Esteban, M.A., J. Xu, J. Yang, M. Peng and D. Qin et al., 2009. Generation of induced pluripotent stem cell lines from tibetan miniature pig. J. Biol. Chem., 284: 17634-17640.

Esteban, M.A., T. Wang, B. Qin, J. Yang and D. Qin et al., 2010. Vitamin $C$ enhances the generation of mouse and human induced pluripotent stem cells. Cell Stem Cell, 6: 71-79.

Feng, Q., S.J. Lu, I. Klimanskaya, I. Gomes and D. Kim et al., 2010. Hemangioblastic derivatives from human induced pluripotent stem cells exhibit limited expansion and early senescence. Stem Cells, 28: 704-712.

Fusaki, N., H. Ban, A. Nishiyama, K. Saeki and M. Hasegawa, 2009. Efficient induction of transgenefree human pluripotent stem cells using a vector based on Sendai virus, an RNA virus that does not integrate into the host genome. Proc. Jpn. Acad. Ser. B Phys. Sci., 85: 348-362.

Hanna, J., S. Markoulaki, P. Schorderet, B.W. Carey and C. Beard et al., 2008. Direct reprogramming of terminally differentiated mature B lymphocytes to pluripotency. Cell, 133: 250-264.

Hu, B.Y., J.P. Weick, J. Yu, L.X. Ma, X.Q. Zhang, J.A. Thomson and S.C. Zhang, 2010. Neural differentiation of human induced pluripotent stem cells follows developmental principles but with variable potency. Proc. Natl. Acad. Sci., 107: 4335-4340.

Jia, F., K.D. Wilson, N. Sun, D.M. Gupta and M. Huang et al., 2010. A nonviral minicircle vector for deriving human iPS cells. Nat. Methods, 7: 197-199.

Kim, J.B., H. Zaehres, G. Wu, L. Gentile and K. Ko et al., 2008. Pluripotent stem cells induced from adult neural stem cells by reprogramming with two factors. Nature, 454: 646-650.

Loh, Y.H., S. Agarwal, I.H. Park, A. Urbach and H. Huo et al., 2009. Generation of induced pluripotent stem cells from human blood. Blood, 28: 5476-5479. 
Nakagawa, M., M. Koyanagi, K. Tanabe, K. Takahashi and T. Ichisaka et al., 2008. Generation of induced pluripotent stem cells without Myc from mouse and human fibroblasts. Nat. Biotechnol., 26: 101-106.

Okita, K., T. Ichisaka and S. Yamanaka, 2007. Generation of germline-competent induced pluripotent stem cells. Nature, 448: 313-317.

Silva, J., O. Barrandon, J. Nichols, T. Theunissen, J. Kawaguchi and A. Smith, 2008. Promotion of reprogramming to ground state pluripotency by signal inhibition. PLoS Biol., 6: e253-e253.

Sommer, C.A., A.G. Sommer, T.A. Longmire, C. Christodoulou and D.D. Thomas et al., 2010. Excision of reprogramming transgenes improves the differentiation potential of iPS cells generated with a single excisable vector. Stem Cells, 28: 64-74.

Stadtfeld, M., K. Brennand and K. Hochedlinger, 2008. Reprogramming of pancreatic $\beta$ cells into induced pluripotent stem cells. Curr. Biol., 18: 890-894.

Tadtfeld, M., M. Nagaya, J. Utikal, G. Weir and $\mathrm{K}$. Hochedlinger, 2008. Induced pluripotent stem cells generated without viral integration. Science, 322: $945-949$.

Takahashi, K. and S. Yamanaka, 2006. Induction of pluripotent stem cells from mouse embryonic and adult fibroblast cultures by defined factors. Cell, 126: 663-676.
Takahashi, K., K. Okita, M. Nakagawa and S. Yamanaka, 2007. Induction of pluripotent stem cells from fibroblast cultures. Nat. Protoc., 2: 3081-3089.

Takahashi, K., M. Narita, M. Yokura, T. Ichisaka and S. Yamanaka, 2009. Human induced pluripotent stem cells on autologous feeders. PloS One, 4: e8067-e8067.

Tchieu, J., R. Jaenisch, N. Maherali, R. Sridharan and W. Xie et al., 2007. Directly reprogrammed fibroblasts show global epigenetic remodeling and widespread tissue contribution. Cell Stem Cell, 1: 55-70.

Warren, L., P.D. Manos, T. Ahfeldt, Y. Loh and H. Li et al., 2010. Highly efficient reprogramming to pluripotency and directed differentiation of human cells using synthetic modified mRNA. Cell Stem Cell, 7: 618-630.

Wernig, M., A. Meissner, J.P. Cassady and R. Jaenisch, 2008. c-Myc is dispensable for direct reprogramming of mouse fibroblasts. Cell Stem Cell, 2: 10-12.

$\mathrm{Yu}$, J., K. Hu, K. Smuga-Otto, S. Tian, R. Stewart, I.I. Slukvin and J.A. Thomson, 2009. Human induced pluripotent stem cells free of vector and transgene sequences. Science, 324: 797-801.

Zhou, H., S. Wu, J.Y. Joo, S. Zhu and D.W. Han et al., 2009. Generation of induced pluripotent stem cells using recombinant proteins. Cell Stem Cell, 4: 381-384.

Zhou, W. and C.R. Freed, 2009. Adenoviral gene delivery can reprogram human fibroblasts to induced pluripotent stem cells. Stem Cells, 27: 2667-2674. 